\title{
Morphological Studies of Wobbler Mouse Dorsal Root Ganglia Show Neurofilamental Disorders
}

\author{
Bastian Ott ${ }^{1}$, Carolin Dahlke ${ }^{1}$, Darius Saberi ${ }^{1}$, Beate Brand-Saberi ${ }^{2}$, Veronika Matschke ${ }^{1}$, Thomas Schmitt-John ${ }^{3}$ and Carsten \\ Theiss $^{1^{*}}$ \\ ${ }^{1}$ Department of Cytology, Institute of Anatomy, Ruhr-University Bochum, Universitätsstraße 150, 44801 Bochum, Germany \\ ${ }^{2}$ Department of Anatomy and Molecular Embryology, Institute of Anatomy, Ruhr-University Bochum, Universitätsstraße 150, 44801 Bochum, Germany \\ ${ }^{3}$ Department of Molecular Biology and Genetics, Neurogenetics, University of Aarhus, C.F. MøllersAllé 3, 8000 Aarhus, Denmark
}

Correspondence to:

Dr. Carsten Theiss

Institute of Anatomy, Department of Cytology

Ruhr-University Bochum, Universitätsstraße 150

D-44780 Bochum, Germany

Tel: (+49) (0)234 32-24560

E-mail: carsten.theiss@rub.de

Received: November 25, 2016

Accepted: February 04, 2017

Published: February 06, 2017

Citation: Ott B, Dahlke C, Saberi D, BrandSaberi B, Matschke V, et al. 2017. Morphological Studies of Wobbler Mouse Dorsal Root Ganglia Show Neurofilamental Disorders. J Neurol Exp Neurosci 3(1): 1-7.

Copyright: (C) 2017 Ott et al. This is an Open Access article distributed under the terms of the Creative Commons Attribution 4.0 International License (CC-BY) (http://creativecommons. org/licenses/by/4.0/) which permits commercial use, including reproduction, adaptation, and distribution of the article provided the original author and source are credited.

Published by United Scientific Group

\begin{abstract}
Amyotrophic lateral sclerosis (ALS) is a fatal neurodegenerative disease with three described forms: The abundant, sporadic ALS (sALS) with approximately $90 \%$ of cases, the familial ALS (fALS) with $5-10 \%$, and the very rare juvenile ALS (jALS) group, which is statistically less relevant. The wobbler mouse, a model for sALS, has been in the focus of research for many decades. Due to symptoms strongly resembling the human ALS pathology, the $\alpha$-motor neurons $(\alpha \mathrm{MN})$ have received the most attention. With regard to pathological cellular processes, particularly those of impaired axonal transport, neuronal tissues in general should be examined. Dorsal root ganglia (DRG) cells are equipped with extremely long axons. Thus, we expected them to be an excellent target for analyzing the cellular mechanisms underlying the disease. In this study, an analysis of the distribution of heavy neurofilaments $(\mathrm{NfH})$ in the perikarya and peripheral nerves of dorsal root ganglia cells from wobbler mice was performed. Here, we demonstrate that sensory neurons are also affected in wobbler mice during the progression of the disease, showing signs of degeneration like those described in the $\alpha \mathrm{MN}$. Furthermore, a highly impaired distribution of neurofilaments and a high number of phosphorylated heavy neurofilaments ( $\mathrm{pNfH}$ ) were observed, not only in large light neurons (LLN), but also in the small dark neurons (SDN) of wobbler mouse DRGs. The accumulation of $\mathrm{pNfH}$ in DRG as well as the loss of $\mathrm{NfH}$ in their axons are promising links to studies promoting high levels of $\mathrm{pNfH}$ in the cerebrospinal fluid (CSF) as an early hallmark of ALS in humans.
\end{abstract}

\section{Keywords}

Dorsal root ganglia, Amyotrophic lateral sclerosis, Wobbler, Neurofilaments, Neurodegeneration

\section{Introduction}

Neurological diseases, and in particular motor neuron diseases (MND), have a great impact on a patient's quality of life. ALS is a common MND with an incidence of 1-3 cases per 100.000 per year and a prevalence of 3-8 per 100.000 , with men being more often affected than women. The average survival is 3 years after the onset of ALS symptoms, typically between the ages of 55 to 60 [1]. Many pathological mechanisms have already been well investigated in animal models with ALS-like phenotypes, however comparison to the human ALS pathology is difficult. A treatment substantially modifying the diseases progression is still lacking. The well-known degeneration of the upper and lower motor neurons are common in the murine models as well as in humans. This 
degeneration results in the denervation of skeletal muscles and eventually to progressive paralysis leading to respiratory arrest. Since first being described by Charcot in 1896, ALS has been investigated intensively and many cellular defects found in animal models have been suggested to cause these symptoms, such as oxidative stress due to mitochondrial dysfunction, protein aggregation, neuroinflammation in different parts of the central nervous system and impaired axonal transport [26]. Up till now, none of the cellular defects found have led to a strategy to modify disease progression in animal models or humans. After decades of ALS research there is still a gap between animal models and humans.

The wobbler mouse is an animal model of importance since it is considered as a model for sALS, representing approximately $90 \%$ of all ALS cases [7-9], although the wobbler mutation does not seem to be a common reason for ALS $[10,11]$. Palmisano and co-workers described enlarged vesicles in wobbler mice $\alpha$-motor neurons $(\alpha \mathrm{MN})$ [12], which indicate an impaired trafficking in wobbler mice, like six out of fourteen human sALS cases. Because of the inherited wobbler mutation, the vacuolar-vesicular protein sorting factor 54 (Vps54) partially loses its function as a subunit of the Golgi-associated retrograde protein (GARP) complex, which is involved in the docking and fusion of endosome-derived vesicles with the trans-Golgi network $[12,13]$. The gene defect is autosomal recessive, with homozygous mice (WR) showing the ALS-like phenotype with a loss of motorneurons in the brain stem, cranial nerve nuclei and spinal cord [14]. Wobbler mice display a wobbling, unsteady gait, and a tremor of the head $[15,16]$ in addition to the progressive paralysis of all four limbs, beginning at the forelegs and proceeding to the hind legs. The different phases and videos of the symptoms were described previously [17].

As a new aspect of research on the wobbler mouse, this study is focused on DRG neurons and their afferent axons. Previously, neurons of the sensory system had not been in focus, due to missing sensory symptoms in ALS patients. Even though morphological alterations in human DRG of ALS cases are well known [18, 19]. Previously, Kawamura et al. [20] provided evidence that large afferent neurons are affected in ALS patients and Heads et al. [21] suggested a more widespread metabolic disturbance in ALS, with parallel involvement of efferent and afferent neurons. The wobbler mutation, as well as the expression of Vps54 is ubiquitous and it is thus to be expected that other neuronal tissues are also affected. Besides that, the anatomical relations between the sensory and the motoric systems make an analogous impairment of both systems appear likely. They have a close relationship within the spinal nerve and the peripheral nervous system, for which intercellular transport mechanisms are crucial to both neuronal cell types, due tothe length of their axons.

In this study, we discovered abnormal accumulations of vesicles in DRG neurons which were used as an indicator for the wobbler defect in $\alpha \mathrm{MN}$ [12]. Furthermore, we observed a significant down-regulation of $\mathrm{NfH}$ gene expression and a redistribution of neurofilaments, with an accumulation of the phosphorylated heavy chain $(\mathrm{pNfH})$ in the perikarya of large light neurons (LLN). So this alteration of $\mathrm{NfH}$ phosphorylation and loss of axonal neurofilaments could be a possible new link to human cases of ALS, since elevated $\mathrm{pNfH}$ levels in the cerebrospinal fluid are currently under debate as a biomarker for ALS [22, 23].

\section{Material and Methods}

All animal experiments in this study have been performed in strict accordance with institutional, German (TschG), and EU guidelines for the care and use of laboratory animals. Breeding and genotyping was done as described earlier in Ott et al. [17]. For all experiments, we used heterozygous (wr/wr) asymptomatic wobbler mice from the pre-symptomatic phase at the age of postnatal day (p) 20 and symptomatic wobbler mice from the evolutionary phase at the age of p40 and age matched controls (wt/wt) from both sexes.

\section{Extraction of neuronal tissues}

Mice were anesthetized, decapitated and fixed though immersion with $2.5 \%(\mathrm{v} / \mathrm{v})$ glutaraldehyde (GA) for electron microscopy or $4 \%(\mathrm{w} / \mathrm{v})$ paraformaldehyde (PFA) for immunohistochemistry. The spinal cord together with the DRGs and the sciatic nerves were dissected en bloc with aid of a binocular microscope, avoiding any traction of the tissues.

\section{Immunohistochemistry}

\section{Specimen preparation}

After fixation for 3 days in 4\% PFA, tissue was transferred into a cryoprotective solution $(30 \%(\mathrm{w} / \mathrm{v})$ sucrose in PBS) for 24 hours. Specimen were separated into DRGs, spinal cord and sciatic nerve and embedded in tissue freezing medium (Cryoglue, SLEE, Mayence, Germany). After freezing, slices were cut with a cryotome (Leica CM 3050 S, Wetzlar, Germany) with a chamber temperature of $-24{ }^{\circ} \mathrm{C}$ and a microscope slide temperature of $-17{ }^{\circ} \mathrm{C}$. Sections between $10 \mu \mathrm{m}$ and $14 \mu \mathrm{m}$ thick were collected on Superfrost Plus (Gerhard Mentzel, Braunschweig, Germany) slides and dried at $37^{\circ} \mathrm{C}$ for 1 hour.

\section{Immunofluorescence staining of $\mathrm{NfH} / \mathrm{pNfH}$}

Permeabilization with $0.3 \%$ (v/v) Triton-X-100 (T8532, Sigma-Aldrich) was performed for $10 \mathrm{~min}$. Anti-NfH antibody, a rabbit polyclonal IgG (RRID: AB_477272; N4142; Sigma-Aldrich, 1:200), was incubated with the sample for 24 hours at $4{ }^{\circ} \mathrm{C}$. After incubation with the primary antibody, blocking was performed with 10\% goat serum (G9023, SigmaAldrich) for $30 \mathrm{~min}$. Labeling was done with anti-rabbit IgG TRITC (RRID: AB_261740; T6778; Sigma-Aldrich, 1:500) through incubation for 2 hours at room temperature. Then the sample was incubated with $\mathrm{pNfH}$ mouse monoclonal $\mathrm{IgG}$ (RRID: AB_306084; ab7795; Abcam, USA, 1:400) again for 24 hours at $4{ }^{\circ} \mathrm{C}$ followed by anti-mouse IgG FITC (RRID: AB_259421; F1010; Sigma-Aldrich, 1:500) for 2 hours at room temperature. Nuclei were stained with DAPI (B2261, Sigma-Aldrich, 1:1000) for 20 min. Every step was followed by 5 times washing with PBS. Finally, slides were covered with fluorescence mounting medium (S3023, Dako). 


\section{Immunofluorescence staining of APP/ RAB7}

Permeabilization was performed with $1 \%$ Triton-X-100 for $10 \mathrm{~min}$. Anti-APP was mouse monoclonal IgG (RRID: AB10718206; SIG39188, Covance, USA), and anti-Rab7 was rabbit polyclonal IgG (RRID: AB_477460; R4779; Sigma-Aldrich, Germany). First, the anti-APP antibody was applied (1:100, 24 hours) followed by intensive washing steps (PBS) and blocking with 10\% goat serum (G9023, Sigma-Aldrich) for $30 \mathrm{~min}$. After two hours incubation with the secondary antibody (anti-mouse TRITC-labelled IgG (RRID: AB_261699; T5393, Sigma-Aldrich, 1:200)), and several washing steps with PBS, incubation with antiRAB7 (1:100, 24 hours) followed. After thorough washing with PBS, anti-RAB7 was then detected by anti-rabbit FITC IgG (RRID: AB_259682; F6005; Sigma-Aldrich, 1:200, 2 hours at room temperature). Finally, slides were covered with fluorescence mounting medium (S3023, Dako). Analysis of all immunohistochemistry experiments was done with the aid of a confocal laser scanning microscope (Zeiss LSM510 Meta), equipped with a Plan-Neofluor 40x/1.3 oil lens and a PlanApochromat 63x/1.4 oil lens.

\section{Electron Microscopy}

After fixation for 3 days in GA, the tissue was washed in $\mathrm{PB}$ for $10 \mathrm{~min}$. Specimen were then incubated with Dalton for $2 \mathrm{~h}$ followed by washing in PB for $10 \mathrm{~min}$. After dehydration in an ascending ethanol series, the tissue was embedded in Epon at a ratio of propylene oxide and Epon, first 3:1 for 45 min, 1:1 for 1 hour then 1:3 for 2 hour, with the last step being pure Epon overnight. Sections were cut with an Ultracut E Reichert-Jung, collected on Formvar coated grids and contrasted with uranyl acetate. Samples were analyzed with a Philips EM 420 (Philips, Holland) transmission electron microscope equipped with a digital CCD camera (Model 792 BioScan; Gatan, USA) and photographic plates system processed with a Ditabis Micron System.

\section{Quantitative Real-Time PCR analysis}

To quantify NFH mRNA levels in dorsal root ganglia taken from the cervical spinal cord of affected p40 mice, total RNA was isolated using ReliaPrep ${ }^{\text {TM }}$ RNA Tissue Miniprep System (Z6110, Promega, Mannheim, Germany) according to the manufacturer's protocol. cDNA synthesis was synthesized from $50 \mathrm{ng}$ of total RNA utilising a QuantiTect ${ }^{\circledR}$ Reverse Transcription Kit (205310, Qiagen, Hilden, Germany). qRT-PCR and cycling conditions were performed using specific oligonucleotide primers (GAPDH forward: GGAGAAACCTGCCAAGTATGA, reverse: TCCTCAGTGTAGCCCAAGA; NFH forward: GACCTGCTCAACGTCAAGAT, reverse: GGGAGTCCTTCAGTAAGAGAGA) using GoTaq $^{\circledR}$ qPCR Master Mix (A6001, Promega, Mannheim, Germany). Reactions were carried out on an CFX Connect ${ }^{\mathrm{TM}}$ Real-Time PCR Detection System (Bio-Rad, Munich, Germany) using 96 well Reaction plates (4ti-0740, 4titude, Berlin, Germany) and Bio-Rad PCR Sealers Microseal 'B' Film Adhesive seal (optically clear; MSB1001, Bio-Rad, Munich, Germany). Melting curves were obtained after each PCR run and showed single PCR products. cDNAs were run in triplicate, controls without reverse transcriptase or without a template were run in duplicates. Expression levels for the gene of interest and for the housekeeping gene GAPDH were measured in three independent PCR runs. Expression ratios were calculated using the geometric mean of the housekeeping gene, GAPDH, to normalize the expression data for the gene of interest according to the $2^{-\Delta \Delta \mathrm{Ct}}-$ method as described by others [24]. qPCR products were additionally analyzed by agarose gel electrophoresis.

\section{Deep-etch freeze-fracture replica}

Rapid-freeze deep-etch electron microscopy was performed as previously described by Meller [25]. Briefly, DRG were frozen using a liquid-propane-jet apparatus (Cryojet QFD 101, Balzers, Lichtenstein). The specimen was then fractured and etched in a Balzers freeze-etching device, with an etching time between 8 and 10 min at $-95^{\circ} \mathrm{C}$ and $8 \times 10^{-7} \mathrm{mbar}$. Platinum was applied at an angle of $35^{\circ}$ to the rotating specimen. The platinum-carbon replicas were cleaned with household bleach, washed in distilled water, and placed unsupported on mesh grids. The pictures of replicas shown are reverse images with the platinum deposits appearing white.

\section{Quantitative analysis}

Statistical analyses of the qPCR data were performed with Prism 5.0 (Graph Pad Inc., La Jolla, USA). Data represent mean values of at least three independent experiments \pm standard error of the mean (SEM). Data were tested for Gaussian distribution by D'Agostino-Pearson tests and experiments were analyzed for significance by unpaired $t$-tests. $\mathrm{P}$-values $\mathrm{p} \leq 0.05$ were considered statistically significant.

For quantification of the amount of $\mathrm{pNfH}$ positive somata within immunofluorescence images, $\mathrm{NfH}$-positive and pNfH-positive somata were counted in DRG of WR and WT. Subsequently, the percentage of $\mathrm{pNfH}$ was calculated for the total number of NfH positive cell somata in the DRG. Somata were considered to be positive at an intensity difference between the background and signal above 300 pixel intensity within a 16-bit image.

For quantification, the neurofilaments within electron microscopic pictures of axon cross-sections were counted. Counted neurofilaments were marked and counted with a click-counter MurGaa Click Counter. Measurements were made with Image J Version 1.49. The collected data were analyzed with Statistica Version 12.7, by StatSoft. A t-test for independent sampling was performed after control of the Gaussian distribution.

\section{Results}

\section{The wobbler defect in DRG neurons}

This study analyses DRG neurons from symptomatic WR mice at $\mathrm{p} 40$. As the progression of symptoms starts at the mouse's forelegs and later proceeds to hind legs, DRGs form cervical andupper thoracic spinal cord were taken for analysis so as to ensure that the material is from anearly affected area. Nevertheless, all wobbler mice used showed partial paralysis 
and wobbling of all four limbs.

Via immunohistochemistry, we could detect accumulations of Rab7 positive vesicles colocalized with the amyloid precursor protein (APP) in wobbler mice (Figure $1 \mathrm{~A}-\mathrm{C}$ ). Rab7 is a small GTPase found in late endosomes and autophagosomes, whereas APP is a cargo protein that is transported through the secretory pathway. Both can be colocalized in vesicles, and this is established as a hallmark for impaired vesicle trafficking in the wobbler mouse [12]. Further transmission electron microscopic (TEM) analysis of DRG neurons additionally revealed high numbers of enlarged vesicles at p40 (Figure 1D; $\mathrm{n}=5$ mice, with every wobbler mouse showing these alterations in DRG neurons). This accumulation is also described in degenerating $\alpha \mathrm{MN}$ of wobbler mice, typically sparing the axon hillock [12, 17]. Together with the APP/Rab7 positive vesicles, this is as a hint for an impaired vesicle trafficking in DRG neurons. At present, this is the first indication of similar neuronal degeneration in DRG neurons as in $\alpha \mathrm{MNs}$.

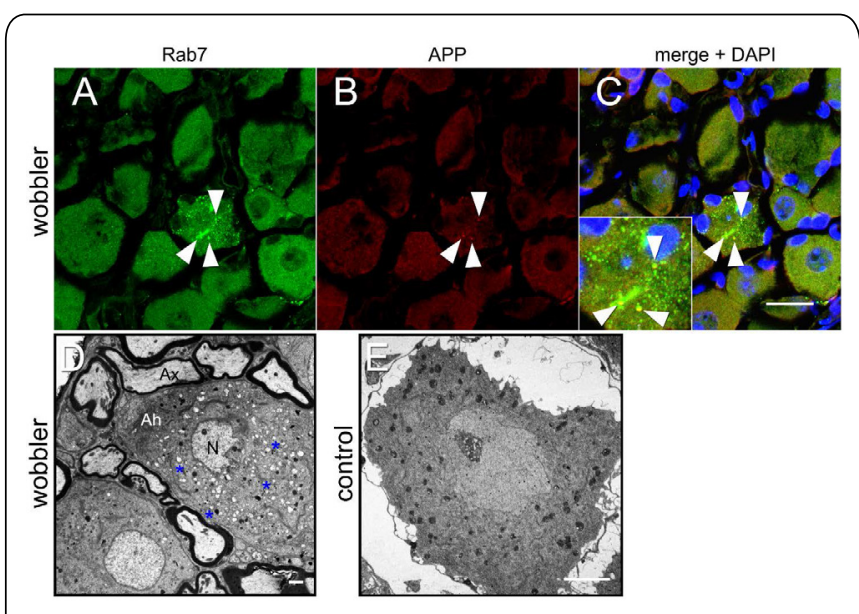

Figure 1: Neurodegeneration in DRG of wobbler mice at $\mathrm{p} 40$.

Immunostaining of an exemplary cervical DRG of symptomatic wobbler mouse at p40 stained against Rab7 (A) (green) as a marker for endosomes and APP (B) (red) as a marker for the exocytotic pathway. Accumulation of Rab7-positive endosomes are demonstrated in A by arrowheads. The merged picture (C) reveals the colocalization with APP, a common sign for the wobbler defect; nuclei are stained with DAPI (blue). Magnification included in C shows colocalizations shining yellow, pointed with arrowheads. Scale bar: $20 \mu \mathrm{m}$. The perikaryon of a random affected sensory neuron at p40 (D) shows vacuolization of soma, (blue stars) sparing axon hillock (Ah) and a degenerating nucleus (N). (E) Sensory DRG neuron from age matched control with many lysosomes, but without vacuolization. Both taken from the cervical spinal cord. Ax = axon; Scale bar: $1 \mu \mathrm{m}$.

\section{Neurofilament disarrangement in the wobbler mouse}

Due to the substantial role of $\mathrm{pNfH}$ in neuronal pathologies, DRG were screened for differences in the distribution of $\mathrm{NfH}$ and $\mathrm{pNfH}$ at $\mathrm{p} 40$. It is well-known that DRG contain different neurons forming a heterogeneous group of subpopulations distinguished by their size, surface molecules, protein content, signaling network, neurotransmitters and receptive characteristics [26, 27]. A basic differentiation can be made by size and their microscopic appearance. As a consequence of different proteins and their density in the soma, small neurons appear dark whereas large neurons appear light. This makes two main groups distinct, the large light neurons (LLN) and the small dark neurons (SDN).
One important difference is the amount of neurofilament, since LLN contain huge amounts of $\mathrm{NfH}$ and the SDN do not $[28,29]$. In WR DRG (Figure 2A) and control DRG (Figure 2G) stained for NfH, no light microscopic differences could be detected. However, quantitative PCR analysis showed a significant down regulation of the $N F H$ gene in DRG in wobbler mice compared to control animals (Figure 2J and 2K). In WR DRG, several neurons stick out with an accumulation of $\mathrm{pNfH}$ (Figure 2B), whereas no $\mathrm{pNfH}$-positive perikarya were detected in controls (Figure $2 \mathrm{H}$ ). In the latter, a strong $\mathrm{pNfH}$-positive signal is clearly seen within the cross sections of the axons. In merged pictures, affected neurons are clearly visible in wobbler mice (Figure 2C), but not in controls (Figure 2I). Quantification of $\mathrm{pNfH}$ positive somata revealed an amount of $11.7 \%$ in WR $(n=6)$, in contrast to no $\mathrm{pNfH}$ positive somata in controls $(n=6)$. Higher magnification of DRG neurons in a wobbler mouse displayed the perinuclear accumulation of $\mathrm{pNfH}$ within the perikaryon of both the LLN and the SDN neuron populations (Figure 2D-F). This aspect is in contrast to the normal distribution of neurofilaments, since SDN normally contain no NfH. The result of an overall disarrangement of neurofilaments is reflected in the transmission electron microscopy (TEM). Whereas mice at p20 do not show a loss or redistribution of neurofilaments in the sciatic nerve (Figure $3 \mathrm{G}$ ), large myelinated nerve fibers in p40 symptomatic WR mice and WT siblings show great differences in neurofilament distribution. WT mice present a well-organized distribution of neurofilaments and microtubules (Figure 3A-C). In contrast, cross and longitudinal sections of large myelinated axons of WR mice show a disorganization of the microtubules and neurofilament networks, as well as a lower density of neurofilaments (Figure $3 \mathrm{D}$ and $3 \mathrm{E})$. High magnifications revealed a loosened structure accompanied by missing crosslinking elements between neurofilaments in WR (Figure 3F). With the aid of deep-etch freeze-fracture, replicas of longitudinal sections of WT DRG axons can be found. These show the typical strict parallel order of neurofilaments, stabilized through numerous cross-linking elements (Figure $3 \mathrm{H}$ ), whereas in WR axons, a disorganization of the neurofilaments is accompanied by a loss of cross-linking elements (Figure 3I). Quantitative analyses revealed a significant loss of neurofilaments in WR axons compared to WT axons $(\mathrm{n}=10 ; \mathrm{p}<0.001)$ (Figure 3J). The mean neurofilament density counted in cross sections in WR is $482 \mathrm{Nf} / \mu \mathrm{m}^{2}$ whereas in WT its $1241 \mathrm{Nf} / \mu \mathrm{m}^{2}$. The diameter of the evaluated axons identified them as A $\alpha$ fibers, which belong to the proprioceptive system. They conduct the sensory information of muscle spindles and Golgi tendon organs. A satisfying answer of whether this is a result of the denervation or a parallel process cannot be given right now. This result emphasizes the hypothesis of an overall disarrangement of the neurofilaments.

\section{Discussion}

In our present study, we detected sensory neurons in the dorsal root ganglia that accumulate APP/Rab7 positive vesicles. During the maturation of autophagosomes and endosomes, the small GTPase Rab7 plays a key role 


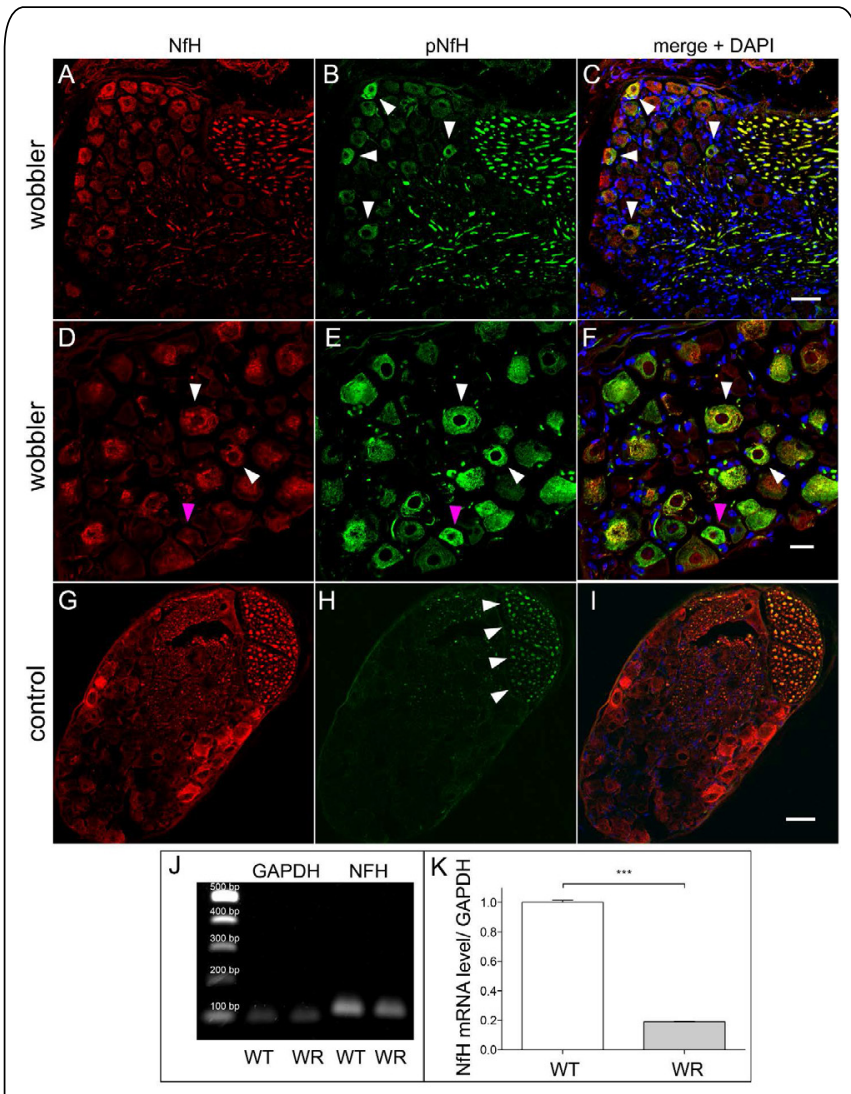

Figure 2: $\mathrm{NfH}$ and $\mathrm{pNfH}$ distribution in the $\mathrm{DRG}$ of wobbler and control mice at $\mathrm{p} 40$.

(A-F) Wobbler mouse (WR) cervical DRG at p40 and (G-I) age matched wildtype mouse (WT) DRG showing $\mathrm{NfH}$ (red) and phosphorylated $\mathrm{NfH}$ (green) distribution. (B, C) WR mouse presents accumulations of $\mathrm{pNfH}$ in several perikarya marked with arrow heads. $(\mathbf{H})$ Whereas in WT mice, $\mathrm{pNfH}$ are restricted to cross-sections of the axons (pointed with arrow heads). (DF) Higher magnification of WR DRG neurons (E) show that perinuclear accumulation of $\mathrm{pNfH}$ (green) is not only restricted to LLN (D-F) (white arrow heads) (D-F) but rather can also be found in SDN (magenta arrow heads). Nuclei are stained with DAPI (blue). Scale bars: A-C and G-I $=50 \mu \mathrm{m}, \mathrm{D}-\mathrm{F}=$ $20 \mu \mathrm{m}$. (J) Exemplary photograph of ethidium-bromide-stained 1\% agarose gel with qPCR products for GAPDH (product length $105 \mathrm{bp}$ ) and NFH (product length $122 \mathrm{bp}$ ) of WT and WR mice. (K) mRNA expression of neurofilament $\mathrm{H}$ in cervical dorsal root ganglia of clinically affected p40 WR versus WT. Expression was determined by quantitative PCR. WR displayed decreased expression of NfH. Data are shown as mean $\pm \mathrm{SEM}$ and are normalized to WT values. Data were tested for significance using the $t$-test. ${ }^{* * *} \mathrm{p}<0.0001 ; 3$ animals per group, $\mathrm{n}=5-8 \mathrm{DRG}$ per animal.

[30]. Amyloid precursor protein APP is a ubiquitous transmembrane protein that is especially expressed in neurons. Processed by $\alpha$ - or $\beta$-secretase, it is the precursor of the wellknown $\beta$-amyloid, which plays a role in Alzheimer's diseases. APP also plays a role in the SOD-1 mouse, a murine model for familial ALS [31]. These vesicles, which neither clearly belong to the exocytotic nor to the endocytotic pathway, are a hallmark of the wobbler phenotype at the subcellular level. They are well described in $\alpha \mathrm{MN}$ s of the spinal cord of wobbler mice [12]. The detection of these vesicles in sensory neurons presents a new aspect,particularly as it has been postulated that the sensory system is not affected. Nevertheless, sensory symptoms are not typical for human ALS and have notyet been tested in wobbler mice. However, a minor influence on the sensory system at the cellular level does not necessarily present with symptoms.

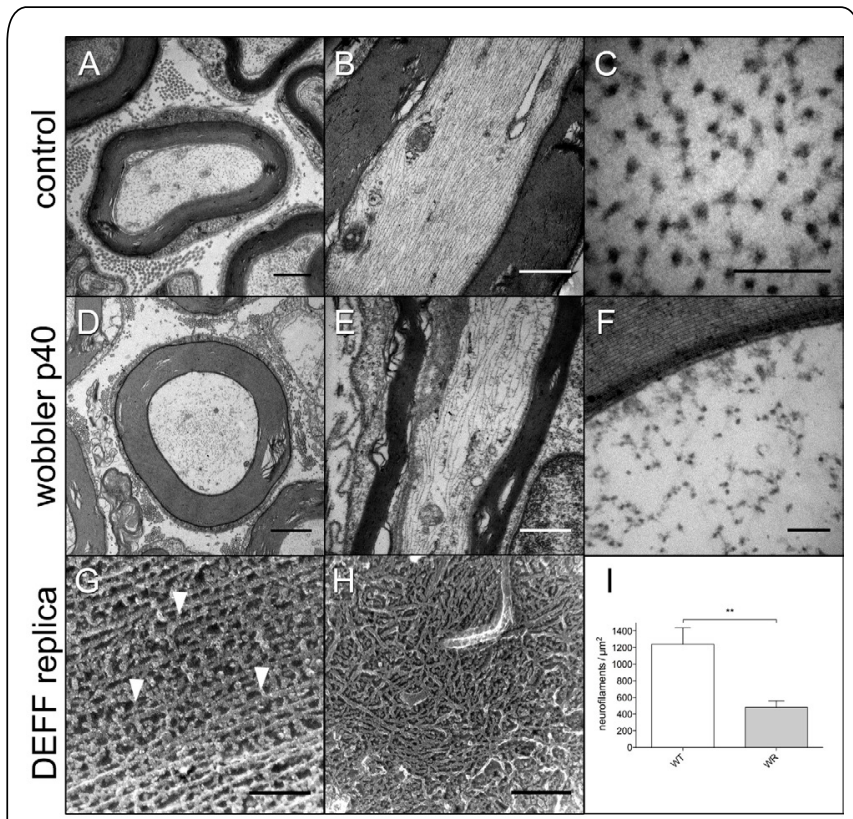

Figure 3: Electron microscopy of wobbler and wildtype sciatic nerve axons at p40.

(A-C) WT show typically parallel arranged neurofilaments in axons of a sciatic nerve at $\mathrm{p} 40$. (D-F) Whereas sections from a WR sciatic nerve at $\mathrm{p} 40$ reveal a loss and disorder of neurofilaments. Higher magnifications of WT (C) and WR mouse (F) reveal few cross-linkers between neurofilaments in WR. The average diameter of WR axons was $17 \mu \mathrm{m}$ and in WT axons $18 \mu \mathrm{m}$, identifying them as $\mathrm{A} \alpha$ fibres. Axons shown are exemplary. Quantitative analysis of neurofilaments in sciatic nerve $A \alpha$ fibresrevealed a significantly reduced number in WR compared to WT $(\mathrm{I} ; \mathrm{n}=10)$. Data are provided as means \pm SEM. Data were tested for significance using Student's t-test. Significant differences are indicated by ${ }^{* * *}$ p $<0.0001$. Deep-etch freeze-fracture electron microscopy shows a pronounced cytoskeletal disorganization with a decreased number of crosslinking proteins in WR $(\mathbf{H})$ versus WT $(\mathbf{G})$. In control cross-linking proteins are indicated by arrow heads.

Scale bars: A, B, D, E $=600 \mathrm{~nm} ; \mathrm{C}, \mathrm{F}=100 \mathrm{~nm} ; \mathrm{G}=550 \mathrm{~nm} ; \mathrm{H}=120 \mathrm{~nm}$ $\mathrm{I}=100 \mathrm{~nm}$.

Immunostaining for phosphorylated and unphosphorylated neurofilaments showed many neurons from both subpopulations in the DRG with an abnormal accumulation of the phosphorylated form in the perikarya, accompanied by an overall loss of neurofilaments and their structure, revealed by TEM. This visible loss of neurofilaments is probably the consequence of a downregulated gene expression. Alterations in neurofilament distribution have been in the focus of research of many neuronal diseases and play a role as a marker of neuroaxonal pathology. For multiple sclerosis, high serum titers of $\mathrm{pNfH}$ correlate with higher lesion volumes in T2 MRT scans and higher Multiple Sclerosis Severity Scores MSSS [32]. But these markers are not only used as prognostic factors in degenerative diseases, but also as markers for acute encephalitis. Here, high neurofilament levels within the cerebrospinal fluid (CSF) are positively correlated with a poor outcome [33]. Moreover, increased perikaryal immunoreactivity of $\mathrm{pNfH}$ in ventral horn cells obtained from patients with ALS was demonstrated [34]. Later, Pugdahl et al. [35] stated that patients with sensory nerve abnormalities represent a variant of ALS, leading to the assumption of many variants in ALS displayinga related phenotype with similar or different cellular disorders. 
Overall, neurofilaments are of major interest for the investigation of neuronal degeneration, but have recently also been discussed as clinical markers for ALS. NfH and especially the phosphorylated form $\mathrm{pNfH}$ seem to play a key role in human sALS. High levels of $\mathrm{pNfH}$ in CSF have been described as a biomarker for sALS $[22,23]$. Plasma NfH levels and disease progression show a close correlation [36].

The new data from this study gives an insight into neurofilament dysregulation in the wobbler mouse, an important animal model for ALS. Even though the link between high levels of $\mathrm{pNfH}$ in the CSF and lower levels in the neuronal tissues could not yet functionally be proven, it is conceivable that there is a release of $\mathrm{pNfH}$ from degenerating neurons.

In addition to this, SOD-1 mice also show accumulations of highly phosphorylated $\mathrm{NfH}$ in neurons as well as misfolded proteins in DRG [37]. This underlines the importance of a closer analysis of neurofilament disorders in ALS as has already been stated by Pernas-Alonso et al. [14] and Julien et al. [38]. In 1999, Julien proposed a neuroprotective function of an accumulation of heavy neurofilaments. In this study it was shown that in SOD-1 mice, a 65\% prolonged overall survival was due to an overexpression of human $\mathrm{NfH}$ [39]. The accumulation and overexpression of $\mathrm{NfH}$ in affected neurons could reflect a protective reaction of these cells to stabilize their perikaryon. Although the neuroprotective function of $\mathrm{NfH}$ could not yet be proven, this aspect could give a link to phosphorylation levels, as phosphorylation also regulates transport and stabilization of the neurofilaments themselves [40]. In further investigations downstream effects of an altered neurofilament distribution in regard to glia activation and neuroinflammation should be addressed. However, until now, no comparable effects could be found in wobbler mice.

Within the wobbler mouse, the changed neurofilament distribution in sensory neurons is a completely new aspect. Besides this, the slow progression gives new insights into the complex process of ALS progression and suggests unknown mechanisms that can slow down the disease. This could be crucial for further steps towards understanding sALS and possible modification of sALS progression.

\section{Acknowledgments}

The authors want to thank Claudia Grzelak and Anke Lodwig for the excellent technical assistance as well as A. Lenz for secretarial work. We also thank D. Terheyden-Keighley for helpful critical discussions. B. Ott owes special thanks to the Stiftung Begabtenfoerderung berufliche Bildung SBB for financial support by providing a scholarship.

\section{References}

1. Wood-Allum C, Shaw PJ. 2010. Motor neurone disease: a practical update on diagnosis and management. Clin Med (Lond) 10(3): 252-258. doi: 10.7861/clinmedicine.10-3-252

2. Ferri A, Cozzolino M, Crosio C, Nencini M, Casciati A, et al. 2006. Familial ALS-superoxide dismutases associate with mitochondria and shift their redox potentials. Proc Natl Acad Sci U S A 103(37): 1386013865. doi: 10.1073/pnas.0605814103

3. Alami NH, Smith RB, Carrasco MA, Williams LA, Winborn CS, et al. 2014. Axonal transport of TDP-43 mRNA granules is impaired by ALS-causing mutations. Neuron 81(3): 536-543. doi: 10.1016/j. neuron.2013.12.018

4. Tadic V, Prell T, Lautenschläger J, Grosskreutz, J. 2014. The ER mitochondria calcium cycle and ER stress response as therapeutic targets in amyotrophic lateral sclerosis. Front Cell Neurosci 8: 147. doi: 10.3389/fncel.2014.00147

5. Dahlke C, Saberi D, Ott B, Brand-Saberi B, Schmitt-John T, et al. 2015. Inflammation and neuronal death in the motor cortex of the Wobbler mouse, an ALS animal model. J Neuroinflammation 12: 215. doi: 10.1186/s12974-015-0435-0

6. Saberi D, Ott B, Dahlke C, Matschke V, Schmitt-John T, et al. 2016. The spatiotemporal pattern of degeneration in the cerebellum of the wobbler mouse. J Neuropathol Exp Neurol 75(4): 347-357. doi: 10.1093/ jnen/nlw005

7. Resch K, Korthaus D, Wedemeyer N, Lengeling A, Ronsiek M, et al. 1998. Homology between human chromosome 2p13.3 and the wobbler critical region on mouse chromosome 11: comparative high-resolution mapping of STS and EST loci on YAC/BAC contigs. Mamm Genome 9(11): 893-898. doi: 10.1007/s003359900890

8. Schmitt-John T, Drepper C, Mussmann A, Hahn P, Kuhlmann M, et al. 2005. Mutation of Vps54 causes motor neuron disease and defective spermiogenesis in the wobbler mouse. Nat Genet 37(11): 1213-1215. doi: $10.1038 / \mathrm{ng} 1661$

9. Moser JM, Bigini P, Schmitt-John T. 2013. The wobbler mouse, an ALS animal model. Mol Genet Genomics 288(5-6): 207-229. doi: 10.1007/ s00438-013-0741-0

10. Corrado L, Gagliardi S, Carlomagno Y, Mennini T, Ticozzi N, et al. 2011. VPS54 genetic analysis in ALS Italian cohort. Eur J Neurol 18(4): 41-42. doi: 10.1111/j.1468-1331.2010.03260.x

11. Meisler M H, Russ C, Montgomery K T, Greenway M, Ennis S, et al. 2008. Evaluation of the Golgi trafficking protein VPS54 (wobbler) as a candidate for ALS. Amyotroph Lateral Scler 9(3): 141-148. doi: 10.1080/17482960801934403

12. Palmisano R, Golfi P, Heimann P, Shaw C, Troakes C, et al. 2011. Endosomal accumulation of APP in wobbler motor neurons reflects impaired vesicle trafficking: implications for human motor neuron disease. BMC Neurosci 12: 24. doi: 10.1186/1471-2202-12-24

13. Karlsson P, Droce A, Moser JM, Cuhlmann S, Padilla CO, et al. 2013. Loss of vps54 function leads to vesicle traffic impairment, protein missorting and embryonic lethality. Int J Mol Sci 14: 10908-10925. doi: 10.3390/ijms140610908

14. Pernas-Alonso R, Perrone-Capano C, Volpicelli F, Porzio U D. 2001. Regionalized neurofilament accumulation and motoneuron degeneration are linked phenotypes in wobbler neuromuscular disease. Neurobiol Dis 8(4): 581-589. doi: 10.1006/nbdi.2001.0403

15. Falconer DS. 1956. Wobbler (wr). Mouse News Lett 15: 23.

16. Duchen LW, Strich SJ. 1968. An hereditary motor neurone disease with progressive denervation of muscle in the mouse: the mutant 'wobbler'. $J$ Neurol Neurosurg Psychiatry 31(6): 535-542.

17. Ott B, Dahlke C, Meller K, Napirei M, Schmitt-John T, et al. 2015. Implementation of a manual for working with wobbler mice and criteria for discontinuation of the experiment. Ann Anat 200: 118-124. doi: 10.1016/j.aanat.2015.03.007

18. Murayama S, Bouldin T, Suzuki K. 1991. Onion bulb formation in the initial complex of neurons in human dorsal root ganglion: their significance and alterations in amyotrophic lateral sclerosis. Acta Neuropathol 82: 462-470. doi: 10.1007/BF00293380

19. Sasaki S, Horie Y, Iwata M. 2007. Mitochondrial alterations in dorsal root ganglion cells in sporadic amyotrophic lateral sclerosis. Acta Neuropathol 114(6): 633-639. doi: 10.1007/s00401-007-0299-1

20. Kawamura Y, Dyck PJ, Shimono M, Okazaki H, Tateishi J, et al. 1981. Morphometric comparison of the vulnerability of peripheral motor and sensory neurons in Amyotrophic Lateral Sclerosis. J Neuropathol Exp 
Neurol 40: 667-675. doi: 10.1007/s00401-007-0299-1

21. Heads T, Pollock M, Robertson A, Sutherland W, Allpress S. 1991. Sensory nerve pathology in amyotrophic lateral sclerosis. Acta Neuropathol 82: 316-320. doi: 10.1007/BF00308818

22. Ganesalingam J, An J, Bowser R, Andersen PM, Shaw CE. 2013.pNfH is a promising biomarker for ALS. Amyotroph Lateral Scler Frontotemporal Degener 14(2): 146-149. doi: 10.3109/21678421.2012.729596.

23. Gonçalves M, Tillack L, de Carvalho M, Pinto S, Conradt H, et al. 2015. Phosphoneurofilament heavy chain and N-glycomics from the cerebrospinal fluid in amyotrophic lateral sclerosis. Clin Chim Acta 438: 342-349. doi: 10.1016/j.cca.2014.09.011

24. Pfaffl MW. 2001. A new mathematical model for relative quantification in real-time RT-PCR. Nucleic Acids Res 29(9): e45. doi: 10.1093/ nar/29.9.e45

25. Meller K. 1989. Chromatolysis of dorsal root ganglion cells studied by cryofixation. Cell Tissue Res 256(2): 283-292. doi: 10.1007/BF00218885

26. Wood JN. 2004. Recent advances in understanding molecular mechanisms of primary afferent activation. Gut 53 Suppl 2: ii9-12. doi: 10.1136/gut.2003.033423

27. Chang-Lin L, Kai-Cheng L, Dan W, Yan C, Hao L, et al. 2016. Somatosensory neuron types identified by high-coverage single-cell RNA-sequencing and functional heterogeneity. Cell Res 26(1): 83-102. doi: $10.1038 / \mathrm{cr} .2015 .149$

28. Lawson SN, Harper AA, Harper EI, Garson JA, Anderson BH. 1984. A monoclonal antibody against neurofilament protein specifically labels a subpopulation of rat sensory neurones. J Comp Neurol 228(2): 263272. doi: 10.1002/cne.902280211

29. Lawson SN, Perry MJ, Prabhakar E, McCarthy PW. 1993. Primary sensory neurones: neurofilament, neuropeptides, and conduction velocity. Brain Res Bull 30(3-4): 239-243. doi: 10.1016/03619230(93)90250-F

30. Hyttinen JM, Niittykoski M, Salminen A, Kaarniranta K. 2012. Maturation of autophagosomes and endosomes: a key role for Rab7. Biochim Biophys Acta 1833(3): 503-510. doi: 10.1016/j. bbamcr.2012.11.018

31. Rabinovich-Toidman P, Rabinovich-Nikitin I, Ezra A, Barbiro B, Fogel H, et al. 2015. Mutant SOD1 increases APP expression and phosphorylation in cellular and animal models of ALS. PLoS One 24: 10-11. doi: 10.1371/journal.pone.0143420

32. Gresle M, Liu Y, Dagley L, Haartsen J, Pearson F, et al. 2014. Serum phosphorylated neurofilament-heavy chain levels in multiple sclerosis patients. JNeurol Neurosurg Psychiatry 85(11): 1209-1213. doi: 10.1136/ jnnp-2013-306789

33. Sellner J, Davies N, Howard S, Petzold A. 2014. Neurofilament heavy chain as a marker of neuroaxonal pathology and prognosis in acute encephalitis. Eur J Neurol 21(6): 845-850. doi: 10.1111/ene.12390

34. Itoh T, Sobue G, Ken E, Mitsuma T, Takahashi A, et al. 1992. Phosphorylated high molecular weight neurofilament protein in the peripheral motor, sensory and sympathetic neuronal perikarya: system dependent normal variations and changes in amyotrophic lateral sclerosis and multiple system atrophy. Acta Neuropathol 83(3): 240-245. doi: 10.1007/BF00296785

35. Pugdahl K, Fuglsang-Frederiksen A, de Carvalho M, Johnsen B, Fawcett P, et al. 2007. Generalised sensory system abnormalities in amyotrophic lateral sclerosis: a European multicentre study. J Neurol Neurosurg Psychiatry 78(7): 746-749.

36. Lu C, Petzold A, Topping J, Allen K, Macdonald-Wallis C, et al. 2015. Plasma neurofilament heavy chain levels and disease progression in amyotrophic lateral sclerosis: insights from a longitudinal study. J Neurol Neurosurg Psychiatry 86(5): 565-573. doi: 10.1136/jnnp-2014-307672

37. Sábado J, Casanovas A, Tarabal O, Hereu M, Piedrafita L, et al. 2014. Accumulation of misfolded SOD1 in Dorsal Root Ganglion degenerating proprioceptive sensory neurons of transgenic mice with Amyotrophic Lateral Sclerosis. Biomed Res Int 2014: 852163. doi: $10.1155 / 2014 / 852163$

38. Julien JP, Kriz J. 2006. Transgenic mouse models of amyotrophic lateral sclerosis. Biochim Biophys Acta 1762(11-12): 1013-1024. doi: 10.1016/j. bbadis.2006.03.006

39. Julien J. 1999. Neurofilament functions in health and disease. Curr Opin Neurobiol 9(5): 554-560. doi: 10.1016/S0959-4388(99)00004-5

40. Ackerley S, Thornhill P, Grierson AJ, Brownlees J, Anderton BH, et al. 2003. Neurofilament heavy chain side arm phosphorylation regulates axonal transport of neurofilaments. J Cell Biol 161(3): 489-495. doi: $10.1083 /$ jcb. 200303138 